\title{
CORRESPONDENCE
}

\section{Bronchiectasis-Diagnosis and Treatment}

by Dr. med. Jessica Rademacher, Prof. Dr. med. Tobias Welte in volume 48/2011

\section{Ejaculate in Differential Diagnosis}

It is perhaps worth mentioning that examination of ejaculate can be helpful in the diagnosis of men with bronchiectasis of unknown origin: if they have cystic fibrosis, these patients usually have azoospermia, but unlike patients with Klinefelter syndrome they usually have testes of normal size. In primary ciliary dyskinesia, sperm are either immotile or very poorly motile. Both findings can indicate a targeted molecular genetic diagnosis, especially when there are also questions of family planning or evaluation of the recurrence risk in children. However, it should first be verified whether this diagnosis may give rise to problems of dubious paternity concerning existing children.

DOI: 10.3238/arztebl.2012.0315a

\section{REFERENCES}

1. Rademacher J, Welte T: Bronchiectasis—diagnosis and treatment. Dtsch Arztebl Int 2011; 108(48): 809-15.

Prof. Dr. med. Hans-Dieter Rott

Erlangen, hdrott@gmx.de

Conflict of interest statement

The author declares that no conflict of interest exists.

\section{In Reply:}

Many thanks for your comment on our review article on the diagnosis and treatment of bronchiectasis. We agree that in individual cases examination of ejaculate may provide evidence of underlying illnesses associated with bronchiectasis. In our article we were obliged to limit ourselves to the most important differential diagnoses and to present the main diagnostic procedures. In our opinion, examination of ejaculate is not part of standard diagnosis for this disease. Rather, it should be put to targeted use to answer specific questions, particularly when family planning plays a role.

DOl: 10.3238/arztebl.2012.0315b

\section{REFERENCES}

1. Rademacher J, Welte T: Bronchiectasis—diagnosis and treatment. Dtsch Arztebl Int 2011; 108(48): 809-15.

Dr. med. Jessica Rademacher Prof. Dr. med. Tobias Welte Medizinische Hochschule Hannover Abteilung Pneumologie welte.tobias@mh-hannover.de

Conflict of interest statement

Dr. Rademacher has received honoraria for speaking from Forest and MSD. Professor Welte has received honoraria for acting as an adviser from Novartis, Bayer Pharma, and Gilead. 